\title{
Multishell Diffusion MRI-Based Tractography of the Facial Nerve in Vestibular Schwannoma
}

\author{
(D) M. Castellaro, (D) M. Moretto, (D) V. Baro, (DS. Brigadoi, (D)E. Zanoletti, (D) M. Anglani, (DL. Denaro, (D). Dell'Acqua, (D)A. Landi, \\ (D) F. Causin, (DD. d'Avella, and (D) A. Bertoldo
}

\begin{abstract}
BACKGROUND AND PURPOSE: Tractography of the facial nerve based on single-shell diffusion MR imaging is thought to be helpful before surgery for resection of vestibular schwannoma. However, this paradigm can be vitiated by the isotropic diffusion of the CSF, the convoluted path of the facial nerve, and its crossing with other bundles. Here we propose a multishell diffusion MR imaging acquisition scheme combined with probabilistic tractography that has the potential to provide a presurgical facial nerve reconstruction uncontaminated by such effects.
\end{abstract}

\begin{abstract}
MATERIALS AND METHODS: Five patients scheduled for vestibular schwannoma resection underwent multishell diffusion MR imaging (bvalues $=0,300,1000,2000 \mathrm{~s} / \mathrm{mm}^{2}$ ). Facial nerve tractography was performed with a probabilistic algorithm and anatomic seeds located in the brain stem, cerebellopontine cistern, and internal auditory canal. A single-shell diffusion MR imaging (b-value $=0,1000 \mathrm{~s} / \mathrm{mm}^{2}$ ) subset was extrapolated from the multishell diffusion MR imaging data. The quality of the facial nerve reconstruction based on both multishell diffusion MR imaging and single-shell diffusion MR imaging sequences was assessed against intraoperative videos recorded during the operation.
\end{abstract}

RESULTS: Single-shell diffusion MR imaging-based tractography was characterized by failures in facial nerve tracking ( $2 / 5$ cases) and inaccurate facial nerve reconstructions displaying false-positives and partial volume effects. In contrast, multishell diffusion MR imaging-based tractography provided accurate facial nerve reconstructions ( $4 / 5$ cases), even in the presence of ostensibly complex patterns.

CONCLUSIONS: In comparison with single-shell diffusion MR imaging, the combination of multishell diffusion MR imaging-based tractography and probabilistic algorithms is a more valuable aid for surgeons before vestibular schwannoma resection, providing more accurate facial nerve reconstructions, which may ultimately improve the postsurgical patient's outcome.

ABBREVIATIONS: $\mathrm{dMRI}=$ diffusion MRI; FN = facial nerve; iFOD2 = second-order integration over fiber orientation distributions; MS = multishell; PVE = partial volume effect; SD-STREAM = streamlines tractography based on spherical deconvolution; SS = single-shell; VS = vestibular schwannoma

fo most cases, surgery for vestibular schwannoma (VS) is performed with the objective of total resection and concomitant preservation of facial nerve (FN) functioning. FN dysfunction is, in fact, thought to be a social and medical issue. ${ }^{1}$ FN functioning preservation rates are up to $98 \%$ in small $\mathrm{VSs}^{2}$ but decrease to $50 \%-70 \%$ for large VSs. ${ }^{3}$ Reconstruction of the FN shape and

Received March 4, 2020; accepted after revision May 22.

From the Padova Neuroscience Center (M.C., M.M., R.D., A.L., D.d., A.B.), Department of Information Engineering (M.C., M.M., S.B., A.B.), Academic Neurosurgery, Department of Neurosciences (V.B., L.D., A.L., D.d.), Department of Developmental Psychology (S.B., R.D.), Otolaryngology Unit, Department of Neurosciences (E.Z.), and Neuroradiology Unit (M.A., F.C.,) University of Padova, Padova, Italy.

M. Castellaro and M. Moretto contributed equally to this work.

Please address correspondence to Valentina Baro, MD, Academic Neurosurgery, Department of Neurosciences, University of Padova, Padova, Italy;

e-mail: valentina.baro@unipd.it

http://dx.doi.org/10.3174/ajnr.A6706 course based on diffusion MR imaging (dMRI) is, undoubtedly, an aid to surgery for large VSs, being, however, technically troublesome. FN identification using conventional structural images, even at high resolution, is affected by the presence of CSF, bony structures, and tumor compression and distortion. ${ }^{4} \mathrm{dMRI}$ and tractography are, in fact, the main techniques used to depict white matter tracts. Their use is widespread for preoperative planning in brain tumor surgery and, recently, also in cranial nerve reconstruction. Since its introduction in 2006, the use of diffusion tensor imaging in FN course prediction has dramatically increased. ${ }^{5}$ To date, the consensus that diffusion tensor imaging is a reliable and valid tool to predict the exact course of the FN is large and undisputed, despite the drawbacks and margins for improvement that the most recent literature keeps bringing to the fore. A major technical limitation of single-shell dMRI (SS-dMRI) combined with deterministic tractography is that it is prone to hindrance by 
Table 1: Summary of clinical characteristics and postoperative results of the 5 patients ${ }^{\mathrm{a}}$

\begin{tabular}{|c|c|c|c|c|c|}
\hline & \multicolumn{5}{|c|}{ Patient No. } \\
\hline & 1 & 2 & 3 & 4 & 5 \\
\hline Sex & $\mathrm{F}$ & $\mathrm{F}$ & $\mathrm{F}$ & M & M \\
\hline Age (yr) & 52 & 57 & 64 & 45 & 53 \\
\hline Symptoms & $\begin{array}{l}\text { Deafness, right tinnitus, } \\
\text { dizziness }\end{array}$ & $\begin{array}{r}\text { Right earache, } \\
\text { hearing loss }\end{array}$ & $\begin{array}{r}\text { Tinnitus, right } \\
\text { hearing loss }\end{array}$ & $\begin{array}{l}\text { Tinnitus, right } \\
\text { hearing loss }\end{array}$ & $\begin{array}{l}\text { Tinnitus, left } \\
\text { hearing loss }\end{array}$ \\
\hline Tumor side & Right & Right & Right & Right & Left \\
\hline Tumor volume $\left(\mathrm{mm}^{3}\right)$ & 347.4 & 370.1 & 1941 & 1153 & 460.6 \\
\hline Tumor size $(\mathrm{mm})$ & $15.5 \times 7$ & $15 \times 6.7$ & $22 \times 17$ & $16.2 \times 14.6$ & $15.5 \times 9.8$ \\
\hline $\begin{array}{l}\text { Extrameatal tumor } \\
\text { dimension }(\mathrm{mm})\end{array}$ & $8.36 \times 5$ & $4.3 \times 6.7$ & $17 \times 12$ & $14.6 \times 10.5$ & $9.8 \times 6.6$ \\
\hline Preoperative HB scale ${ }^{b}$ & 1 & 1 & I & 1 & 1 \\
\hline Postoperative HB scale & 1 & I & VI & III-IV & 1 \\
\hline Koos classification ${ }^{c}$ & II & II & III & III & II \\
\hline
\end{tabular}

Note:-HB indicates House and Brackmann.

${ }^{a}$ Both preoperative and postoperative FN functions are reported according to the scale of House and Brackmann. ${ }^{8}$

${ }^{b}$ House and Brackmann scale: I (normal), normal facial function in all areas; III (moderate dysfunction), gross: obvious but not disfiguring difference between 2 sides; noticeable-but-not severe synkinesis, contracture, and/or hemifacial spasm. At rest, normal symmetry and tone. Motion forehead: slight-to-moderate movement. Eye: complete closure with effort. Mouth: slightly weak with maximum effort; IV (moderately severe dysfunction), gross: obvious weakness and/or disfiguring asymmetry. At rest: normal symmetry and tone. Motion forehead: none. Eye: incomplete closure. Mouth: asymmetric with maximum effort; VI (total paralysis), no movement.

c Classification of Koos et al: 9 grade II, small tumor with protrusion into the cerebellopontine angle; no contact with the brain stem; grade III, tumor occupying the cerebellopontine cistern with no brain stem displacement.

the presence of CSF, ${ }^{6}$ yielding partial volume effects that could instead be circumvented using multi-shell dMRI (MS-dMRI). In addition, b-values on the order of $1000 \mathrm{~s} / \mathrm{mm}^{2}$ are suboptimal for resolving fiber bundles with high angular curvature and untangling crossing fibers. ${ }^{7}$ Here, we describe an approach based on MS-dMRI and probabilistic tractography for FN reconstruction specifically devised to overcome these limitations and assess its reliability by evaluating the $\mathrm{FN}$ reconstruction adherence to the anatomic intraoperative videos recorded during the operation. A direct comparison between a standard approach based on SSdMRI and deterministic tractography and the present approach based on the combination of MS-dMRI and probabilistic tractography was performed to provide a quantitative estimate of the relative performance of both approaches in $\mathrm{FN}$ reconstruction.

\section{MATERIALS AND METHODS \\ Patient Population}

We examined 5 consecutive patients with VS (mean age, 54.2 years; range, 45-64 years) scheduled for surgical resection at the University Hospital of Padova. The female/male ratio was 1.5:1, and the mean VS volume was $854.4 \mathrm{~mm}^{3}$ (range, 347-1941 $\mathrm{mm}^{3}$ ). The main symptoms at presentation were hearing loss and tinnitus, and all patients showed a preserved FN function according to the scale of House and Brackmann. ${ }^{8}$ VS dimensions were classified according to Koos et al; ${ }^{9}$ in 2 cases, the lesion compressed the brain stem (Table 1). All patients underwent a translabyrinthine approach assisted by intraoperative neuromonitoring of the cranial nerves. A gross total resection was attained in each patient. Each operation was video-recorded as routinely done by our skull base team.

\section{Image Acquisition}

Data acquisition was performed with a 3T Ingenia MR imaging scanner (Philips Healthcare) equipped with a 32-channel head coil. Patients were administered the standard presurgical MR imaging protocol, which included 3D T2-weighted driven equilibrium radiofrequency reset pulse (TR/TE, 1500/241 ms; 8 minutes and 40 seconds, $0.4 \times 0.4 \times 0.6 \mathrm{~mm}$ ) and post-contrastenhanced T1 high-resolution isotropic volume examination (TR/ TE, 5.8/3 ms; 6 minutes and 20 seconds, $0.4 \times 0.4 \times 0.5 \mathrm{~mm}$ ). Patients underwent an MS-dMRI acquisition: dMRI sequences were single-shot EPI-acquired with 2 phase-encoding polarities: anterior-posterior and posterior-anterior. The scanning parameters of the anterior-posterior dMRI sequence (TR/TE, 5408/ $98 \mathrm{~ms}$; voxel size, $2 \times 2 \times 2 \mathrm{~mm}$; FOV, $224 \times 224 \times 80 \mathrm{~mm}$ ) included 4 b-values $\left(0,300,1000,2000 \mathrm{~s} / \mathrm{mm}^{2}\right)$ and a number of $(12,3,64)$ noncollinear directions for diffusion-weighted volumes and 8 repetitions for $b_{0}$, while the posterior-anterior dMRI sequence included 3 b-values $\left(0,300,1000 \mathrm{~s} / \mathrm{mm}^{2}\right)$ and a number of (12-32) noncollinear directions and 8 repetitions for $b_{0}$. The patient's tolerance was a factor considered in the development of the dMRI protocol, for a total acquisition time of 23 minutes and 25 seconds.

\section{dMRI Preprocessing}

Anterior-posterior and posterior-anterior dMRI sequences were merged. Denoising and estimation of the noise level were performed with the MRtrix3 dwidenoise function (https://mrtrix. readthedocs.io/en/latest/dwi_preprocessing/denoising.html), ${ }^{10}$ while motion, distortion, and eddy current correction were performed with FSL topup and eddy tools (https://fsl.fmrib.ox.ac. uk/fsl/fslwiki/topup). ${ }^{11,12}$ Diffusion-weighted MR images were coregistered to the T2-weighted image using the Advanced Normalization Tools Software Registration tool (http://stnava. github.io/ANTs/ $)^{13}$ using an affine transformation.

\section{Fiber-Tracking Analysis on MS Data}

Tractography was performed with MRtrix3, using the multishell multitissue constrained spheric deconvolution method, ${ }^{6}$ exploiting the distribution of fiber orientations for each voxel estimated using the tissue-specific response functions. ${ }^{14}$ The probabilistic secondorder integration over fiber orientation distributions (iFOD2) algorithm ${ }^{15}$ was used for streamline generation (MS-iFOD2). The 
parameters of the algorithm were set as follows: number of streamlines $=500,000$, step size $=1 \mathrm{~mm}$, maximum angle between successive steps $=45^{\circ}$, fractional anisotropy cutoff for terminating tracts $=0.1$, radius of spheric seeds $=2 \mathrm{~mm}$. The minimum and maximum length of the streamlines varied among patients, depending on the individual distance between seeds, thus obtaining a consistent reconstruction of the FN. The spheric seed for initiating the tracking was placed at the origin of the $\mathrm{FN}$ in the internal auditory canal, and 2 additional spherical seeds, placed at the FN passage in the cerebellopontine cistern and at the end in the brain stem, were fed to the algorithm as inclusion ROIs. The 3 anatomic seeds were selected in the T2-weighted image. Two additional exclusion masks were generated, one by segmenting the VS in the T1 space using ITK-SNAP software (www.itksnap.org) ${ }^{16}$ and registering the segmentation mask to the dMRI, and the other one, drawn in the dMRI space, to exclude streamlines representing false-positives. This second mask excluded the temporal regions, the medial and superior part of the pons, the trigeminal nerve, and streamlines that formed nonphysiologic structures, such as loops. Both masks were fed to the iFOD2 algorithm as exclusion regions during streamline generation.

\section{Fiber-Tracking Analysis on SS Data}

To compare the proposed MS-dMRI protocol with a more standard SS-dMRI approach currently used in clinical practice, we repeated tractography using a single shell from the available data, considering dMRI volumes acquired with b-values equal to 1000 $\mathrm{s} / \mathrm{mm}^{2}$ (32 anterior-posterior directions and 32 posterior-anterior directions).

Streamline generation was performed using both a probabilistic and a deterministic algorithm because both approaches were used in previous studies. ${ }^{17,18}$ The iFOD2 algorithm was used for probabilistic tracking (SS-iFOD2), using the same anatomic seeds and parameters of the algorithm as used for MS-dMRI tracking. Streamlines tractography based on spherical deconvolution (SD-STREAM) ${ }^{19}$ was instead used for deterministic streamline generation (SS-SD-STREAM), using the same anatomic seeds and tracking parameters used for the proposed MS-iFOD2 approach. We used the same masks as those used for the MS-iFOD2 for both the SS-iFOD2 and the SS-SD-STREAM tracking, thus masking the temporal regions, the medial superior part of the pons, and the trigeminal nerve, while removing, for each method, nonphysiologic tracts such as loops.

The SD-STREAM algorithm was also run in a different configuration (SS-SD-STREAM-L), using the number of seeds and tracking parameters in a previous analogous work using fibertracking techniques for cranial nerve reconstruction in the presence of VS. ${ }^{17}$ The SS-SD-STREAM-L approach was performed on SS data by setting the turning angle to $30^{\circ}$ and a step size of $0.66 \mathrm{~mm} .{ }^{17}$ The SS-SD-STREAM-L approach was run both by placing a single anatomic seed in the internal auditory canal (as in Yoshino et $\mathrm{al}^{17}$ ) and placing 2 seeds, one in the internal auditory canal and the other one in the brain stem (as in Zolal et $\mathrm{al}^{18}$ ). No mask was used with the SS-SD-STREAM-L approach.
Table 2: Intraoperative location of FN relative to the VS, assessed at the origin of the nerve and in the cisternal passage tract according to the classification of Sampath et $\mathrm{al}^{20}$

\begin{tabular}{lcc}
\hline & \multicolumn{2}{c}{ Intraoperative Findings } \\
\cline { 2 - 3 } Patient & FN Origin & FN Passage \\
\hline 1 & Al & AM \\
2 & Al & AM \\
3 & AM & P \\
4 & AM & AS \\
5 & AM & AS \\
\hline
\end{tabular}

Note:-Al indicates anterior-inferior; AM, anterior-medial; AS, anterior-superior; $\mathrm{P}$, posterior.

\section{Validation of the Fiber Tracts}

To validate the tractography results, we compared the intraoperative position of the FN relative to VS location with that obtained using dMRI-based tractography. The intraoperative position of the FN relative to the VS location was inferred by the medical team from the intraoperative videos of the operation. The method described by Sampath et $\mathrm{al}^{20}$ (ie, partitioning the FN location into anterior, posterior, or polar) was used to classify the FN position relative to VS location, both on the brain stem surface and in the cerebellopontine cistern. This classification was applied to both the intraoperative findings and the tractography reconstructions, in view of a quantitative comparison between the 2 modalities.

\section{Compliance with Ethical Standards}

All procedures were in accordance with the ethical standards of the institutional research committee and with the 1964 Declaration of Helsinki plus later amendments. Informed consent was obtained from each individual patient included in the present study.

\section{RESULTS}

The classification of Sampath et $\mathrm{al}^{20}$ of intraoperative positions of the FN for each patient is reported in Table 2. Three patients (henceforth referred to as patients 1,2, and 5) featured a standard dislocation of the FN caused by VS, so that the 3 individual anatomic seeds were easy to recognize. Patients 3 and 4 were described by the neurosurgeons as extreme cases, based on a very complex pattern of the FN course found intraoperatively. More specifically, the FN of patient 3 was located on the posterior part of the VS, a location associated with $1.3 \%$ occurrence. ${ }^{5}$ In addition, the FN of patient 3 was divided into several thin fibers splayed on the VS capsule devoid of a plane of dissection. During VS resection, the FN was the first structure that the surgeon encountered, identified by electrophysiologic stimulation of the posterior part of the VS. Because of its atypical distortion, the VS could not be removed without scarifying the FN, which was reconstructed using a biologic graft bringing about a postsurgical FN paralysis and consequent indication for rehabilitation. In patient 4, the FN originated in a direction perpendicular to the vestibular nerve, rather than parallel, and was frayed into many fibers. Tractography results obtained using each protocol and tracking method are summarized in Table 3 and succinctly described in the forthcoming paragraphs. For each patient, the 
Table 3: Results of the fiber-tracking reconstructions obtained with the 4 adopted approaches for each patient

\begin{tabular}{|c|c|c|c|c|c|}
\hline \multirow[b]{2}{*}{ Patient } & \multirow[b]{2}{*}{ MS-iFOD2 } & \multirow[b]{2}{*}{ SS-iFOD2 } & \multirow[b]{2}{*}{ SS-SD-STREAM } & \multicolumn{2}{|c|}{ SS-SD-STREAM-L } \\
\hline & & & & 1 Anatomic Seed & 2 Anatomic Seeds \\
\hline 1 & Accurate & $\begin{array}{l}\text { Different nerve } \\
\text { reconstruction }\end{array}$ & Algorithm failure & Accurate, presence of FP & Accurate \\
\hline 2 & Accurate & $\begin{array}{l}\text { Accurate, presence } \\
\text { of PVE }\end{array}$ & Accurate & Accurate, presence of FP and PVE & $\begin{array}{l}\text { Accurate, presence } \\
\text { of FP }\end{array}$ \\
\hline 3 & Partially accurate & $\begin{array}{l}\text { Partially accurate, } \\
\text { presence of FP }\end{array}$ & Algorithm failure & $\begin{array}{l}\text { Partially accurate, presence of FP } \\
\text { and PVE }\end{array}$ & $\begin{array}{l}\text { Inconsistent } \\
\text { reconstruction }\end{array}$ \\
\hline 4 & Accurate & $\begin{array}{l}\text { Accurate, presence } \\
\text { of PVE }\end{array}$ & Algorithm failure & Inconsistent reconstruction & Algorithm failure \\
\hline 5 & Accurate & Algorithm failure & Algorithm failure & Inconsistent reconstruction & Algorithm failure \\
\hline
\end{tabular}

Note:-FP indicates false-positives.

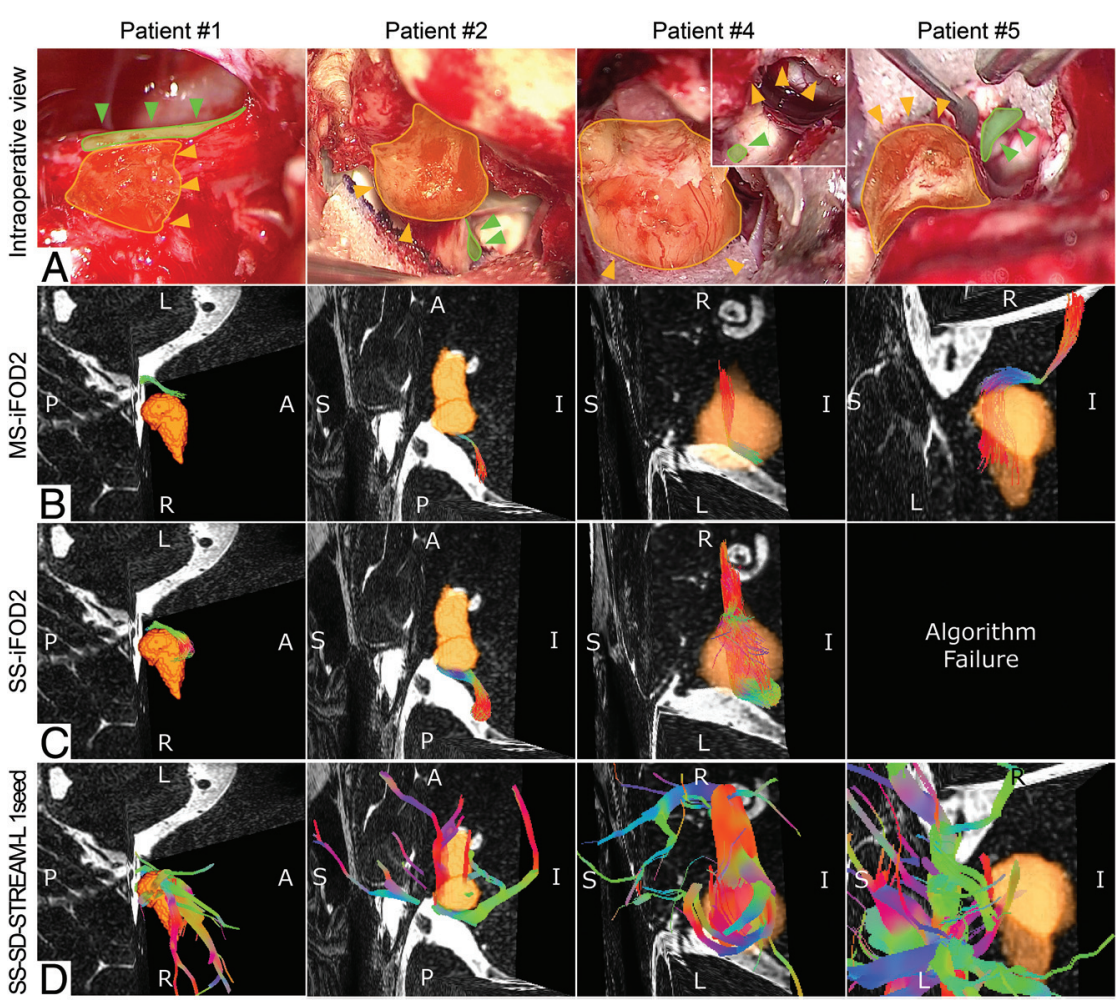

FIG 1. Each column shows a specific patient, from top to bottom. A, The intraoperative view of the VS (yellow area) with the position of the VS highlighted (orange arrows) and the position (green arrows) of the FN (green area). B, C, and D, MS-iFOD2, the SS-iFOD2, and the SS-SDSTREAM-L with 1 anatomic seed tractography reconstruction of the $F N$ with a $3 D$ reconstruction of the VS (orange) displayed in the same orientation as in the intraoperative view, respectively. $A$, For patient 4, two images of the VS, before and after its debulking, highlight the residual tumor capsule (orange arrows) and the FN (green arrow).

reconstruction adherence of the tracking to the intraoperative video of the operation is reported.

\section{Patient 1}

MS-iFOD2 tracking confirmed the intraoperative course of the FN (Fig $1 B$ ), while SS-iFOD2 tracking failed to reconstruct the $\mathrm{FN}$, providing, in place of the $\mathrm{FN}$, a reconstruction of the cochlear nerve (Fig 1C). The SS-SD-STREAM approach resulted in a lack of tracked streamlines. When only 1 seed was used, the SS-SDSTREAM-L approach faithfully reconstructed the FN, showing, however, several false-positive streamlines (Fig 1D). The inclusion of the second seed provided a more accurate reconstruction of the $\mathrm{FN}$ accompanied by a reduction of false-positive tracts.

\section{Patient 2}

Both MS-iFOD2 (Fig $1 B$ ) and SSiFOD2 (Fig 1C) tracking confirmed the intraoperative course of the FN. Compared with MS-iFOD2, SSiFOD2 provided an $\mathrm{FN}$ reconstruction contaminated by partial volume effect (PVE), incorrectly suggesting an altered and enlarged size of the FN. The SS-SD-STREAM tracking faithfully reconstructed the FN. SS-SDSTREAM-L, used in combination with a single seed, faithfully reconstructed the FN course but affected by PVE and false-positives (Fig 1D). However, when 2 seeds were used, both the PVE and the false-positives tracts were reduced to nil.

\section{Patient 3}

The iFOD2 algorithm provided a partially accurate reconstruction; with MS-dMRI-based tractography, this was confined to the FN only (Fig 2B), whereas with the SS-dMRI-based one, it comprised also portions of other nerves (Fig 2C). The SS-SD-STREAM resulted in a lack of tracked streamlines, whereas the SS-SD-STREAM-L using 1 seed yielded multiple intersecting bundles of false-positive tracts, also containing a portion of the FN (Fig 2D). Adding a second seed provided an inaccurate FN reconstruction, consisting of only false-positives.

\section{Patient 4}

Tracking performance bore a high degree of resemblance to that of patient 2 for the iFOD2 algorithm (Fig $1 B,-C$ ). The SS-SDSTREAM approach resulted in a lack of tracked streamlines. SSSD-STREAM-L failed to reconstruct the FN course, with an 

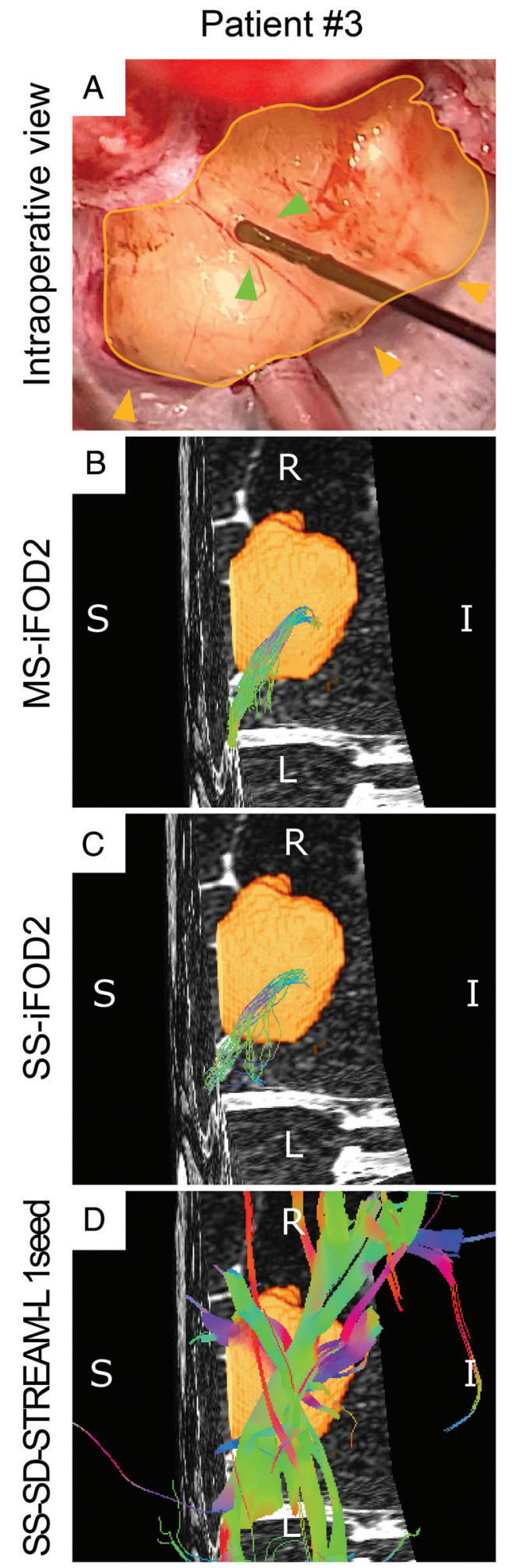

FIG 2. Results for patient 3 (see text for details). A, The intraoperative view of the VS (yellow area) highlighting the position of the VS (orange arrows) and the position of the FN (green arrows). Also shown is the stimulation site suggesting that the FN was frayed and spread on the tumor surface. Note that the FN is posterior compared with the VS position. $B, C$, and $D$, The MS-iFOD2, the SS-iFOD2, and the SS-SDSTREAM-L with 1 anatomic seed tractography reconstruction of the FN with a $3 \mathrm{D}$ reconstruction of the VS (orange) displayed in the same orientation as in the intraoperative view. inaccurate nerve reconstruction when using 1 seed (Fig 1D) and a lack of tracked streamlines when using 2 seeds.

\section{Patient 5}

The course of the FN was reliably reconstructed with the MSiFOD2 tracking (Fig 1D), whereas the other 2 approaches failed to track the FN. SS-iFOD2, in particular, failed to track streamlines (Fig 1C), and the SS-SD-STREAM approach resulted in a lack of tracked streamlines. The SS-SD-STREAM-L approach using a single seed resulted in an inaccurate nerve reconstruction displaying false-positives (Fig 1D). The addition of a second seed discarded the false-positive-tracked nerve, resulting in a lack of tracked streamlines.

\section{DISCUSSION}

Preoperative FN tracking in patients affected by VS could provide essential additional information to surgeons other than the standard structural images. ${ }^{5}$ Although SS-dMRI for the FN course prediction has been shown to approximate, with sufficient precision, the FN anatomic structure, ${ }^{17,18}$ the results of the present study were clear-cut in showing that the joint use of more evolved dMRI acquisition schemes, such as MS sequences, and different tracking algorithms using multi-tissue distribution of fiber orientations outperform SS-dMRI in several respects. Critically, MSdMRI was successful in containing the pervasive detrimental effects caused by the isotropic contribution from the CSF, which usually hinders the FN tractogram.

The results of the present study showed that MS-dMRI-based FN reconstructions bore a high degree of resemblance to the real FN course found intraoperatively in 4 of the 5 patients examined, even in the presence of anatomically peculiar patterns. The partial failure to track the entire FN course in patient 3 can likely be ascribed to the noncoherent structure of the $\mathrm{FN}$, which was frayed and spread over the VS capsule. MR imaging-based tractography relies on the presence of a defined group of myelinated fibers following the same direction. The stopping criteria of a tractography algorithm are based on thresholds in fractional anisotropy and the angle between successive steps. Both criteria prevented the tractography from yielding an accurate reconstruction of the real FN pattern over the VS capsule. Future studies ought to investigate whether improving the resolution and the signalto-noise ratio of the dMRI could overcome this limitation. Note that the apparent failure in the FN reconstruction of patient 3, which was only partially correspondent to the intraoperative findings in every portion of the FN course, could nonetheless have been informative to surgeons about the nonstandard positioning of the FN fibers. Furthermore, the patient could have been appropriately informed about the plausible risk of FN damage before VS surgery.

The SS-iFOD2 tracking provided accurate reconstructions in 2 patients (patients 2 and 4), though characterized by PVE, which affects especially small-fiber reconstruction. This effect can be mitigated using an explicit and separated distribution of fiber-orientation estimation for nerves and CSF, which can be obtained only with the MS approach. ${ }^{6,14}$ MS-iFOD2 provides a better description of the FN when CSF and FN coexist in 1 voxel. In the other 3 patients $(1,3$, and 5), SS-iFOD2 tracking failed to reconstruct any 
nerve or the correct nerve. In patient 1, the SS-iFOD2 tracking provided the reconstruction of the cochlear nerve in place of the FN. This could be explained by the likely inability of the algorithm to distinguish the 2 nerves that run parallel and very close to each other. In our extreme case, instead, although a portion of the FN was present in the reconstruction, it was embedded in several falsepositives. By relying just on this reconstruction, the neurosurgeon could not infer any clear information about the real location of the FN. Finally, in patient 5, SS-iFOD2 completely failed to reconstruct any nerve. This result could be explained by the inability of SS protocols, which use low b-values, to solve high-fiber curvature angles, such as those present in the FN of this patient. These angles could be solved by exploiting higher b-values, as in our proposed MS protocol, which indeed successfully reconstructed the FN.

The SS-SD-STREAM tracking failed to reconstruct any nerve in patients 1,3,4, and 5, and correctly reconstructed the FN of patient 2 only. In patient 2 , the FN showed a modest dislocation and followed a straight path, which could be easily detected by the deterministic algorithm. In patient 1, the failure in FN reconstruction could be ascribed to the short distance of the FN relative to the cochlear nerve and by the inability of the algorithm to reach all the 3 seed points. In patient 3 , the complex structural configuration of the FN was the likely source of the failure in $\mathrm{FN}$ reconstruction, for the algorithm could not distinguish the multiple possible pathways of the FN. In patients 4 and 5, the lack of tracked streamlines could be a consequence of the inability of the SD-STREAM to resolve the pronounced angular curvature of the FN. The SS-SD-STREAM-L tracking provided accurate reconstructions in 2 patients (patients 1 and 2) but was generally affected by PVE. In these patients, its performance was improved (ie, a high decrease of false-positives and PVE) when using 2 seeds rather than just one. In patients 3, 4, and 5, the SS-SDSTREAM-L tracking provided unsuccessful reconstructions. In patient 3 , the approach using a single seed provided a reconstruction comprising many tracts attributable to false-positives and, among these, a partially accurate FN course was identified. However, when using 2 seeds, SS-SD-STREAM-L provided an FN reconstruction completely inconsistent with the intraoperative findings.

The configuration of the FN, frayed in many fibers, was very complex, and the algorithm had too many degrees of freedom. Despite the number of seeds used, the reconstruction would not have been informative to the neurosurgeon. In patients 4 and 5 , SS-SD-STREAM-L tracking failed to reconstruct any of the correct nerves. This failure could be explained by the very high angular curvature of the FN. The angle of curvature allowed in the SSSD-STREAM-L approach was set to $30^{\circ}$ as in Zolal et al, ${ }^{18}$ and this value may have been too small to allow the algorithm to correctly reconstruct the FN tract from the origin to the ending seed. Relying on these reconstructions, the neurosurgeon could be completely misled about the real location of the FN.

The results obtained using the SS protocol, commonly used in clinical practice, show that the reliability of the FN reconstruction could be quite low, above all when difficult cases are under investigation. Therefore, we invite neurosurgeons to pay maximum attention to the reliability of tractography reconstructions produced by standard approaches.
This study had limitations, owing to the small sample size and a prolonged acquisition time compared with standard SS-dMRI. On the hypothesis of using this protocol as a preoperative planning protocol, rather than a diagnostic tool, the total acquisition time could be a less limiting factor. A range of possible solutions could, on the other hand, be adopted to shorten the acquisition time, such as the use of the simultaneous multisection technique $^{21}$ or a reduction of the acquisition subset used for distortion correction. Future studies should validate the use of these possible strategies in FN tracking in patients with VS.

\section{CONCLUSIONS}

The results of this study showed that the use of an MS-dMRI protocol for a probabilistic tracking of the FN course could be a powerful aid for a better presurgical planning of VS resection, compared with the routinely used SS-dMRI protocols. An accurate reconstruction of the entire course of the FN was possible only applying the probabilistic tractography algorithm on the acquired MS-dMRI data and using anatomic information obtained from structural MR images (ie, 3 seeds of passage of the FN, indicated by an expert neuroradiologist). Furthermore, the tractography analyses not only reconstructed a faithful FN course but also provided valuable information about the anatomy of the FN in 2 patients described as limited cases for the complex pattern of the FN fibers. Therefore, this more accurate patient-specific information, provided by the MS-dMRI-based tractography analysis, could possibly decrease the duration of an operation, help the surgeons to preserve the $\mathrm{FN}$, and improve postsurgical patient outcomes.

\section{REFERENCES}

1. Samii M, Gerganov VM, Samii A. Functional outcome after complete surgical removal of giant vestibular schwannomas. $J$ Neurosurg 2010;112:860-67 CrossRef Medline

2. Mazzoni A, Zanoletti E, Denaro L, et al. Retrolabyrinthine meatotomy as part of retrosigmoid approach to expose the whole internal auditory canal: rationale, technique, and outcome in hearing preservation surgery for vestibular schwannoma. Oper Neurosurg (Hagerstown) 2018;14:36-44 CrossRef Medline

3. Youssef AS, Downes AE. Intraoperative neurophysiological monitoring in vestibular schwannoma surgery: advances and clinical implications. Neurosurg Focus 2009;27:E9 CrossRef Medline

4. Sartoretti-Schefer S, Kollias S, Valavanis A. Spatial relationship between vestibular schwannoma and facial nerve on three-dimensional T2-weighted fast spin-echo MR images. Am J Neuroradiol 2000;21:810-16 Medline

5. Baro V, Landi A, Brigadoi S, et al. Preoperative prediction of facial nerve in patients with vestibular schwannomas: the role of diffusion tensor imaging-a systematic review. World Neurosurg 2019;125:2431 CrossRef Medline

6. Jeurissen B, Tournier J-D, Dhollander T, et al. Multi-tissue constrained spherical deconvolution for improved analysis of multi-shell diffusion MRI data. Neuroimage 2014;103:411-26 CrossRef Medline

7. Tournier JD, Calamante F, Connelly A. Determination of the appropriate $b$ value and number of gradient directions for high-angularresolution diffusion-weighted imaging. NMR Biomed 2013;26:177586 CrossRef Medline

8. House JW, Brackmann DE. Facial nerve grading system. Otolaryngol Head Neck Surg 1985;93:146-47 CrossRef Medline 
9. Koos WT, Day JD, Matula C, et al. Neurotopographic considerations in the microsurgical treatment of small acoustic neurinomas. J Neurosurg 1998;88:506-12 CrossRef Medline

10. Veraart J, Novikov DS, Christiaens D, et al. Denoising of diffusion MRI using random matrix theory. Neuroimage 2016;142:394-406 CrossRef Medline

11. Andersson JL, Skare S, Ashburner J. How to correct susceptibility distortions in spin-echo echo-planar images: application to diffusion tensor imaging. Neuroimage 2003;20:870-88 CrossRef Medline

12. Andersson JL, Sotiropoulos SN. An integrated approach to correction for off-resonance effects and subject movement in diffusion MR imaging. Neuroimage 2016;125:1063-78 CrossRef Medline

13. Avants BB, Tustison NJ, Song G, et al. A reproducible evaluation of ANTs similarity metric performance in brain image registration. Neuroimage 2011;54:2033-44 CrossRef Medline

14. Dhollander T, Raffelt D, Connelly A. Accuracy of response function estimation algorithms for 3-tissue spherical deconvolution of diverse quality diffusion MRI data. In: Proceedings of the Joint Meeting of the European Society for Magnetic Resonance in Medicine and Biology and the International Society of Magnetic Resonance in Medicine, Paris, France. June 16-18, 2018:1569

15. Tournier J-D, Calamante F. Connelly A. Improved probabilistic streamlines tractography by 2 nd order integration over fibre orientation distributions. In: Proceedings of the International Society for Magnetic Resonance in Medicine, Stockholm, Sweden. May 6-13, 2010:1670

16. Yushkevich PA, Piven J, Hazlett HC, et al. User-guided 3D active contour segmentation of anatomical structures: significantly improved efficiency and reliability. Neuroimage 2006;31:1116-28 CrossRef Medline

17. Yoshino M, Kin T, Ito A, et al. Combined use of diffusion tensor tractography and multifused contrast-enhanced FIESTA for predicting facial and cochlear nerve positions in relation to vestibular schwannoma. J Neurosurg 2015;123:1480-88 CrossRef Medline

18. Zolal A, Juratli TA, Podlesek D, et al. Probabilistic tractography of the cranial nerves in vestibular schwannoma. World Neurosurg 2017;107:4753 CrossRef Medline

19. Tournier JD, Calamante F, Connelly A. MRtrix: diffusion tractography in crossing fiber regions. Int J Imaging Syst Technol 2012;22:5366 CrossRef

20. Sampath P, Rini D, Long DM. Microanatomical variations in the cerebellopontine angle associated with vestibular schwannomas (acoustic neuromas): a retrospective study of 1006 consecutive cases. J Neurosurg 2000;92:70-78 CrossRef Medline

21. Setsompop K, Cohen-Adad J, Gagoski BA, et al. Improving diffusion MRI using simultaneous multi-slice echo planar imaging. Neuroimage 2012;63:569-80 CrossRef Medline 